\title{
Novel Scale Development for Fear of Falling and Falls: Analysed Using a Semiparametric Ratio Estimator (SPRE)
}

\author{
Deborah Weissman-Miller ${ }^{1} \&$ Kay C. Graham ${ }^{1}$ \\ ${ }^{1}$ Occupational Therapy, Brenau University, Gainesville, Georgia, USA \\ Correspondence: Deborah Weissman-Miller, Occupational Therapy, Brenau University, Gainesville, GA, USA. \\ E-mail: dweissman-miller@brenau.edu
}

Received: June 15, 2015 Accepted: July 8, 2015 Online Published: July 21, 2015

doi:10.5539/ijsp.v4n3p161

URL: http://dx.doi.org/10.5539/ijsp.v4n3p161

\begin{abstract}
Fear of falling (fof) and falls are increasingly severe worldwide public health problems. The Falls Weight Function (FWF) uses a new scale to incorporate fear of falling (fof) into analyses with participants who have already experienced a fall. FWF is a weight function in the semiparametric ratio estimator (SPRE) to predict a change point and point estimations. FWF data is a discrete set of numbers that is finite or countable. In a study using Stepping On $囚$ fall prevention program, initial data from fof responses were counted, summed as increments of .10 values (ranging from .1 to 1.0), and then multiplied by 1 fall. The un-weighted value of 1 fall was multiplied by the weight function for fof. Then, the scale of falls and fof is the same, and represented on a continuum from fear of falling to having a fall, so that all participants can be treated and analyzed together.
\end{abstract}

Keywords: Fear of falling, (fof), weight function, Falls Weight Function (FWF), semiparametric ratio estimator (SPRE), falls

\section{Introduction}

\subsection{Introduce the Problem}

Analysis of fall prevention outcomes is complicated by the heterogeneity of the aging population (Tinetti, De Leon, Doucette, \& Baker, 1994) and the variations in outcome measures (Jørstad, Hauer, Becker, \& Lamb, 2005). While researchers have utilized both fear of falling (fof) and actual fall incidence as outcome measures, often the nuanced effects of a program are excluded from research studies because fof cannot be figured into actual fall outcomes. The new Falls Weight Function (FWF) uses a new scale developed to enable researchers to incorporate fear of falling (fof) into analyses with participants who have already experienced a fall. The FWF is applied during data structuring in Excel so that it will be applied throughout the regression analyses of the semiparametric ratio estimator (SPRE) for single-subjects and small $n$ designs to predict outcomes from change points in data series.

Why are we interested in predicting health outcomes based on fear of falling and falls in older adults? High fear, high number of falls and low self efficacy can cause restrictions in activity and quality of life (Jørstad et al., 2005; Tinetti, De Leon, Doucette, \& Baker, 1994). Though prevalence of fof varied greatly due to measure variablity, a systemic review identified major fof risk factors as having a fall, being female, and aging adults (Scheffer, Schuurmans, van Dijk, van der Hooft, \& de Rooij, 2008). Consequences of fof have been identified as falls, decreased fall-related self efficacy, and activity restriction/decreased quality of life (Jørstad et al., 2005; Scheffer et al., 2008; Talley, Wyman, Gross, Lindquist, \& Gaugler, 2014; Tinetti et al., 1994; Tinetti, Richman, \& Powell, 1990). As a result of its negative influence and impact on falls and function in older adults, those with fof should be addressed within fall prevention programming. Why is this problem important?

Falls, fear of falling and fall related self-efficacy should be considered as related yet separate behavioral constructs which can negatively impact older adults (Jørstad et al., 2005; Talley et al., 2014). As a result, many fall prevention programs address fallers, those at risk for falls and those with a fear of falling using the same content; however, related constructs are typically measured separately. Research on the effectiveness of program content in fall prevention should effectively address the older adult population across all of these constructs. The difficulty lies in methods to analyze this diverse population in statistically sound methodology.

The separation of constructs in fall prevention research has resulted in the exclusion of those with fof from standard fall remediation research (Clemson et al., 2004). A new measurement strategy utilizing a Falls Weight Function (FWF) allows analysis incorporating both fof and falls to better identify effective intervention strategies. 


\subsection{Background}

America is growing older. The percentage of 65 and older adults in the US is projected to increase from $13 \%$ of the population in 2012 to $21 \%$ of the population while those 85 and over will triple in number by the year 2040 (Profile of older americans: 2013, 2013). Increasing age has been associated with increasing fall risk and risk of injury due to falls (Centers for Disease Control and Prevention [CDC], 2010; Lawlor, Patel, \& Ebrahim, 2003). The numbers are startling. In a three month survey, $15 \%$ of older adult respondents reported falling at least once with injuries reported by $31 \%$ of those who had fallen (Stevens, Corso, Finkelstein, \& Miller, 2006). In addition to increased risk of injury and death due to falls, fear of falling has also been associated with avoiding activities, decreased quality of life, and increased depression (Arfken, Lach, Birge, \& Miller, 1994; Schepens, Sen, Painter, $\&$ Murphy, 2012; Tinetti et al., 1994). Fear of falling increases with age, gender (women), and fraility in the older adult population (Arfken et al., 1994). Researchers need research methods which can account for the complex issues faced by older adults in relation to falls and fear of falling.

A recent study of fof over a two year period identified that new onset fof and fof changes over time may have different risk factors (Talley et al., 2014). The participants' baseline fof as measured by balance confidence using the Activities-specific Balance Confidence Scale (ABC) was independently associated with lower scores on the SF36 physical function and mental function scales while decreasing balance confidence over time (5\% average decrease) was associated with worsening strength, balance, activity, and social network levels (Talley et al., 2014). These authors cautioned that "researchers including fear of falling as a secondary outcome in fall prevention trials need to make careful decisions about who to include in the study and how to measure fear of falling" (p. 630) due to conflicting construct definitions and variability in fof measures. The Falls Weight Function (FWF) offers a statistical approach to include in analysis both those with fof and those who have fallen. This is especially important for multiple factorial fall prevention programs such as Stepping On® (Clemson, 2009; Clemson et al., 2004) which address those with fof and/or a history of falls for "at risk" community dwelling older adults.

\subsection{Pilot Studies}

Participants with concern or fear of falling, a history of falls or both were included in both the original RCT fall intervention study by Clemson (2009) as well as during the recent pilots using SPRE analysis methods following provision of Stepping On®, a 7 week standardized fall prevention program. As a multifactorial fall prevention program, Stepping On® addresses both fof and fall incidence; it is designed to improve self-efficacy or confidence to prevent falls, reduce incidence of falls, and facilitate ability to identify fall risk behaviors and how to mitigate this risk by behavioral choices and skills (Clemson et al., 2004). Participants who were community dwelling older adults showed up to a 30\% reduction in fall rate following the program (Clemson et al., 2004; Stevens, 2010). In the original Clemson (2004) program, participants with fof were included in the program and in the fall self-efficacy analysis, but analysis of changes to fof could not be made within analysis of fall reduction. The Falls Weight Function (FWF) was conceived to provide a statistical method to include participants with fof and actual falls into the same analysis. This was first used during a series of pilot studies (with full IRB approval) exploring the use of SPRE analysis method with small " $n$ " designs. Ahmad, Lesko, Marslander and Randall (2014) analyzed data from a pilot study using the new SPRE analysis model that analyses user response data for each individual and then the mean response of 3 participants at any point in time. Occupational therapists implemented the Stepping Onß program for 22 initial participants and collected weekly self-ratings using the Activities-specific Balance Confidence (ABC) Scale (Powell \& Myers, 1995). The 7 data points collected weekly from the $\mathrm{ABC}$ were interpolated to provide the required 13-14 data points per participant for the SPRE model. This interpolation was first used in a pilot study by Byrd, A.C., Goodbar, H.A., Lesley, W.N., Martin, C.C. (2013) and so the Ahmad et al. (2014) analysis computed and compared three individual point estimates whose p-values were statistically significant with $\mathrm{p} \leq 0.05$, to the mean data starting from a change point derived from initial data in the first 7.0 weeks. This analysis was chosen to illustrate the statistical use of the FWF in SPRE for fall prevention in older adults because little statistical analytical work has been published which looks at prevalence and incidence of adults with fof together with those who have fallen. Even if, as Talley, et al. (2014) suggested, fear of falling and having a fall are separate constructs, they can both be considered at the same time like fruit in a bowl by using FWF. This concept was first presented at the Joint Statistical Meeting by Weissman-Miller \& Graham (2014).

\section{Method}

In many cases, statistical estimations can be made from initial data from a least squares regression, or by predicting a trend line, or in time series forecasting when the use of a model to forecast future events is based on known past events. However, health outcomes are often in the form of an exponential cumulative distribution of a survival 
function, when residuals are not distributed normally (Cleves, Gould, \& Gutierrez, 2004) and for time-to-eventdata (Hosmer, Lemeshow, \& May, 2008) or a parametric Weibull cumulative distribution where past performance may be unavailable or not relevant to the disease outcomes being measured. Therefore, the SPRE model has been developed to derive a 'change point' where the participant adapts to the therapy, and to predict long-term outcomes in the form of a Weibull distribution using initial data from the patient(s) in a clinical trial or in therapy.

\subsection{Assumptions for Data Using the Falls Weight Function (FWF)}

The first assumption is that fof data is taken from a 10-16 point survey questions of falls concern for a participant during different activities. The second assumption is that in a discrete setting, a weight function is a positive function defined on a discrete set A, which is finite or countable. A third assumption is that in fear of falling (fof), the weight function is defined as positive and countable.

\subsection{Assumptions for the Data Using the FWF in the SPRE Model}

The first assumption is that approximately 13-14 data points in the primary trial follows an initial quasi-linear form that can be analyzed by ordinary least squares to develop a change point. This is true for a single subject or a small group where the mean indicates the response to treatment. The second assumption is that the outcome values are ordered data. If the data are not approximately linear, then transforms were used to linearize the data; finally, values on the time axis are ordered together with the ordered outcome data. A third assumption is that a change point can be determined from initial data before an arbitrary cutoff in time or treatment numbers. A fourth assumption is that long-term data outcomes can be predicted from the change-point using the ratio of the point estimation function (Weissman-Miller, 2012) times the prior outcome prediction as given by Weissman-Miller (2013).

\subsection{Derive the Falls Weight Function}

For a real value function, the un-weighted sum of ' $\mathrm{f}$ ' on $\mathrm{A}$ is:

$$
\sum_{a \in A} f(a)
$$

A weighted function $\mathrm{w}$ where $\mathbb{R}^{+}$equals a set of positive real numbers is:

$$
A \rightarrow \mathbb{R}^{+}
$$

The weighted sum is then:

$$
\sum_{a \in A} f(a) \cdot w(a)
$$

In this summation, $f(a)=1$ (fall) for this function where 1.0 is the image of 'a' under ' $\mathrm{f}$ '. Then $f(a)=1$ is a partial function with respect to the weighted elements. When the weighted sum is in the numerator of the ratio transform data used in occupational therapy to transform the raw data so that it can be analyzed using the ordinary least squares model (OLS) in SPRE (Weissman-Miller, Shotwell \& Miller, 2012), then the transform for the fof data is given as:

$$
\sqrt{\frac{\sum_{a \in A} f(a) \cdot w(a)}{\text { Measure }}}
$$

In this pilot study the measure used was the activities balance confidence scale (ABC), where the data to be analyzed was given by a ratio as given in equation (4) of the square root of the ratio of the FWF divided by the $\mathrm{ABC}$ scale, or by the number of falls divided by the same scale.

\subsubsection{Weighted and Unweighted Functions}

The $A \rightarrow \mathbb{R}^{+}$is given in this pilot study by the fear of falling fof 'Very Concerned' column of the intake survey. In this study, the un-weighted function of ' $\mathrm{f}$ ' on ' $\mathrm{a}$ ' is given in equation (1). The weighted function in equation (3) is the Falls Weight Function (FWF) where the $f(a)$ equals 1 fall and $w(a)$ is the weighted sum of each survey box checked from 0.10 to 1.00 for each of the 10 items on the survey, which then equal 1 fall. The total of these countable responses is a fraction of all possible responses from 1/10 to 1.00. The $\mathrm{ABC}$ ratio given in equation (4) as the measure is applied to each random variable $\mathrm{X}, \mathrm{Y}$ of the OLS regression because it is a function of the linear transform of the raw data. 


\subsubsection{The Continuum}

Now, fear of falling (fof) is directly compared to 1 fall through the OLS, where both $R^{2}$ and p-values, of the highest or lowest F distribution in the SPRE model, form the change point for each participant. The scale of falls and fof is the same, and represented on a continuum of fear of falling to having a fall, so that all participants can be treated together and analyzed in the same set of linear regressions. The fof-falls scale is now continuous from 0.1 to any number of falls $n$ on the real number line and given as:

$$
0.1,0.2,0.3,0.4,0.5,0.6,0.7,0.8,0.9,1,2,3,4,5, \ldots, n
$$

While this new scale does not mean that the constructs of fof or having a fall are causal, it does mean that they are now shown to be related so that all older adults can now benefit and they may all be treated with the same falls prevention programs - without the exclusion of participants who have not fallen but who have fof.

\subsection{The Change Point in the Initial Patient Least Squares Data for FWF in SPRE}

Generally speaking, change-point regression is a regression problem in which the expected value of the dependent variable or response is assumed to have a different functional form in several neighborhoods of the explanatory variable space according to Khodadadi and Asgharian (2008). In the SPRE model, the determination of the change point is a structural change that for falls shows the dynamic nature of the changes. Details of the methodology are given in Weissman-Miller (2013). The time at the change point is the location of the ratio predictions for long-term estimates, which produces a gradually changing predictive model. This is true for data measured for FWF and falls.

These results were computed by the R Development Core Team (2010), R Commander by Fox (2005) and using a program called SPRE written in R by D. Weissman-Miller (2014) and running in RStudio (2014). It should be noted that in using FWF in this SPRE model, the F statistic is still determined comparatively from the regression analyses, where the $\mathrm{P}$ value is used to determine statistical significance of the regression analysis to that point. The resulting change point, of the highest or lowest $\mathrm{F}$ statistic computed, is then denoted by the value of time, or treatment session number, together with the measured outcome in the dataset.

\section{Results}

A comparison of falls data with fof data is shown in Figure (1). It can be seen that the data are similar in slope although not identical.

\section{RESULTS -Comparative Data a Fall to FoF}
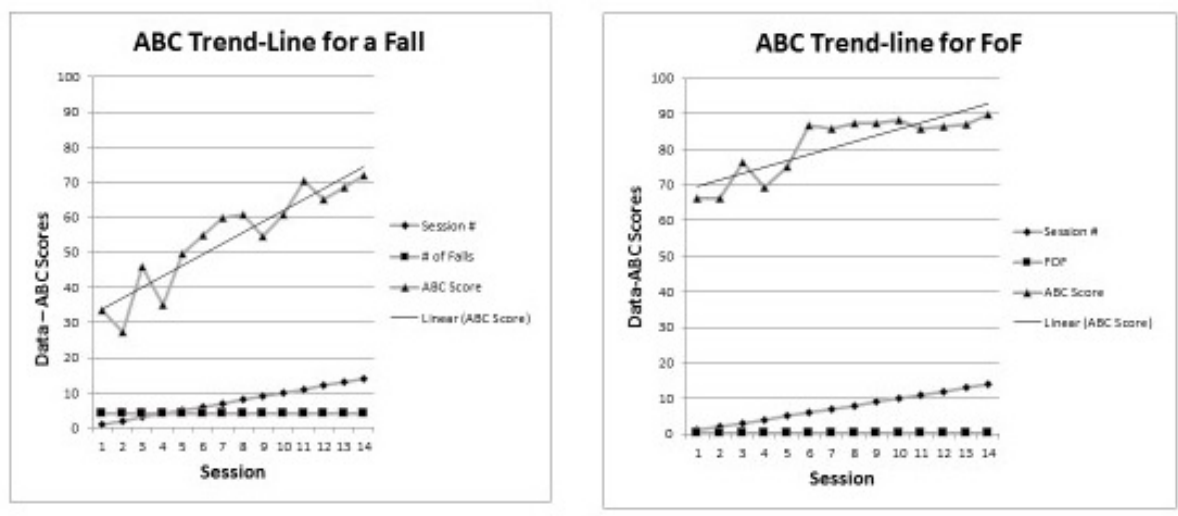

Figure 1. Comparison data for participants with a fall and fof

Furthermore, the graph shows that the participant who had a fall started with a lower balance confidence than the participant with fof. This makes sense when comparing the data side by side. Furthermore, the participant who had sustained a fall increased confidence more than the participant with fof.

As shown in Table (1), there is only one highest F statistic in this dataset at Session 3. In any therapy, the treatment 
should be carried out past a first mode to be sure that all relevant modes, such as a second mode of response, have been recorded.

It can be seen from the analyses in Table (1) that the highest $\mathrm{F}$ statistic is at time $=3$ sessions from the backward stepwise elimination method, including the variables $\mathrm{x}$ (time) and $\mathrm{y}$ (outcome). Each dataset, starting at an assumed full model at 13 sessions from 7 weeks, is analyzed to determine $R^{2}$ and the F statistic. Then the next dataset is analyzed from 13 sessions down to 2 sessions.

In this paper, similar to other research using SPRE, a change point is defined as the outcome and the session number for the highest $\mathrm{F}$ statistic for analysis of the mean, where the participant outcomes with respect to time are no longer linear (Miller, Weissman-Berman, \& Martin, 2008; Weissman-Miller \& Miller, 2011), or the data point at which the character of the regression changes, in general, from linear to the shape of an exponential or the Weibull cumulative distribution (Weissman-Miller, 2010; Weissman-Miller, 2013). Using the SPRE program in this analysis, the residuals at the change points were quasi-random and normal predictions for each of the participants, and where the data were statistically significant at the change point a means analysis was conducted and predictions computed together with the mean of 3 participants as shown in Table (1). The results for all are graphed in Figure (2).

Table 1. Calculation to Determine the Change point for Fof in SPRE - Participant F

\begin{tabular}{|c|c|c|c|c|c|c|}
\hline \multicolumn{5}{|c|}{ Change Point Calculations: \# Falls of FoF/ABC } & \multicolumn{2}{|c|}{ Predictions } \\
\hline Session & $R^{2}$ & F-Statistic & p-value & OrdsqrtABC & Session & Prediction No. \\
\hline 5 & & & & .0502 & 1 & .05101022 \\
\hline 4 & 1.0 & & & .0506 & 2 & .05101041 \\
\hline $3^{*}$ & .9999 & 19680.0 & .004538 & .05101 & 3 & .05101056 \\
\hline 6 & .01244 & .0252 & .8885 & .05103 & 4 & .05101067 \\
\hline 13 & .25 & .9998 & .391 & .05121 & 5 & .05101075 \\
\hline 9 & .3108 & 1.804 & .2504 & .05164 & 6 & .05101082 \\
\hline 12 & .4506 & 4.101 & .09873 & .05165 & 7 & .05101088 \\
\hline 8 & .3873 & 3.792 & .09942 & .05175 & 8 & .05101092 \\
\hline 7 & .2931 & 2.903 & .1322 & .05186 & 9 & .05101096 \\
\hline 10 & .3331 & 3.996 & .08064 & .05186 & 10 & .05101099 \\
\hline 11 & .3906 & 5.769 & .03978 & .05208 & 11 & .0510102 \\
\hline 2 & .04236 & 1.018 & .3369 & .05223 & 12 & .05101105 \\
\hline 1 & .01506 & .1682 & .6896 & .02345 & 13 & .05101107 \\
\hline
\end{tabular}

In the means analysis, the SPRE model predicts skill improvement via the Stepping On $₫$ falls prevention treatment. Participant $d$ had a fall prior to the program, while participants $f$ and $p$ had fear of falling (fof) which were analyzed using the FWF. The relative error of point estimates to the primary data is excellent for treatment where there was an early individual change point and subsequent data from the pilot study. The predictions track through the test data. The SPRE ratio predictions from each change point (the initial data prediction) show that there is significant improvement from the start of the trial to the change point for these 3 participants. The SPRE analysis confirms the same result measured in each participant for which there is an early change point.

The predictions in this paper are based on the calculation of individual change points for 3 participants where the change point of the means analysis are based on the values of previous events. Using SPRE, the means of 3 or more participants whose results are statistically significant, are analysed to predict the response of other (larger) similar populations. For this reason, this model is especially useful in predicting the response to treatment for individuals who have had a least 1 fall or who have a fear of falling fof. Furthermore, predicting the numbers of treatments until the participant becomes stable reduces waste and controls costs in health care. Talley et al. (2014, p.631) discussed 
the problem attending to individual change patterns in their analysis: "this analysis examined average change; however, each participant has a different pattern of change. Future research could select only those women who demonstrate decreasing confidence and identify what predicts change for these women to identify low confidence early and to intervene to arrest its decrease." This can now be done using SPRE with the determination of a 'change point' for each participant.

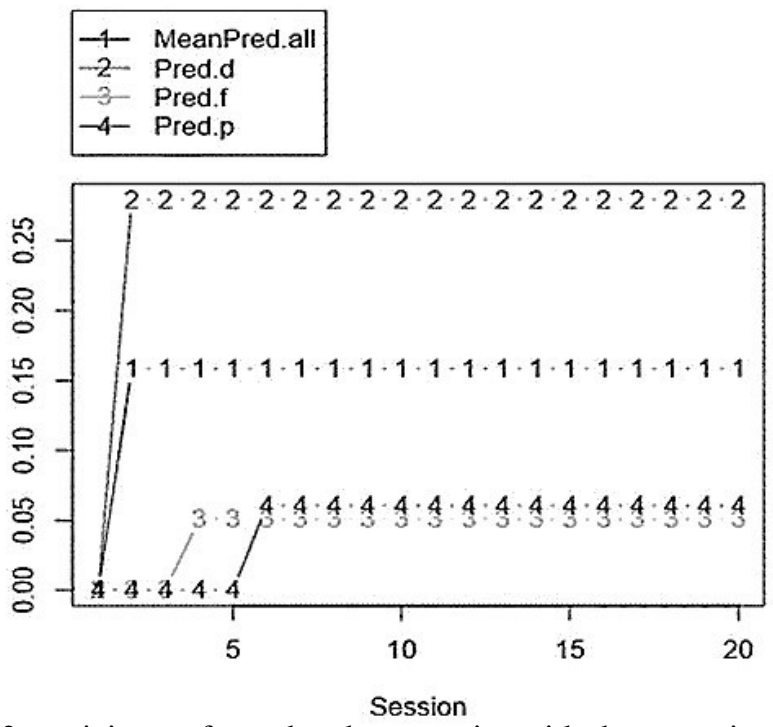

Figure 2. Point estimates of 3 participants from the change point with the mean in the Falls Prevention program as measured by the ABC measure. (Used with permission from Ahmad, S., Lesko, B., Marslender, A., Randall, S. (2014))

\section{Discussion}

The point estimates can be analyzed for participants who have had one or more falls and, using FWF, can also be analyzed for participants who have varying levels of fear of falling. Statisticians can now analyse participants who have fear of falling in the same groups and with the same treatments (such as Stepping On $($ ) that are used in fall prevention for elders. This enables both groups to regain confidence and further participate in activities meaningful to them which in turn improves their health. The graphs are very similar for the long-term test data for treatment of individuals who have had a fall or who have a fear of falling (fof), as shown in Figure (2). This point estimator from the SPRE ratio using FWF or the number of falls has also been shown to be consistent at the upper and lower bounds of prediction and robust for single subjects or a small group (Weissman-Miller, 2013). Furthermore, the absolute bias ratio of this SPRE ratio will be typically small in practice as given in Meng (1993), whether the model is for a small group or the long-term predicted outcomes for a single subject. If the small group has 3-10 participants, then it is proposed that inferences may be made to a similar, larger population provided that the $\mathrm{p}$ value at the change point is $\leq 0.05$. Finally, these two groups can be analyzed together using the semiparametric ratio (SPRE) which allows for heterogeneity in single-subject or small group design.

\section{References}

Ahmad, S., Lesko, B., Marslender, A., Randall, S. (2014). The effectiveness of the Stepping On® program in reducing the fear of falling and incidence of falls in the independent community-dwelling older adult population. (Unpublished master's thesis). Brenau University, Gainesville, GA.

Arfken, C. L., Lach, H. W., Birge, S. J., \& Miller, J. P. (1994). The prevalence and correlates of fear of falling in elderly persons living in the community. American journal of public health, 84(4), 565-570.

Byrd, A.C., Goodbar, H.A., Lesley, W.N., Martin, C.C. (2013). Effectiveness of the Stepping On Program in reducing the fear of falling and the incidence of falls in community-dwelling adults. (Unpublished master's thesis). Brenau University, Gainesville, GA.

Centers for Disease Control and Prevention [CDC]. (2010). Web-based injury statistics query and reporting system(wisqars) [database online]. Available from URL: www.cdc.gov/ncipc/wisqars

Clemson, L. (2009). Preventing falls in the elderly using "stepping on": A group-based education program Inter- 
national handbook of occupational therapy interventions (pp. 465-471): New York, NY: Springer.

Clemson, L., Cumming, R. G., Kendig, H., Swann, M., Heard, R., \& Taylor, K. (2004). The effectiveness of a community-based program for reducing the incidence of falls in the elderly: A randomized trial. Journal of the American Geriatrics Society, 52(9), 1487-1494.

Cleves, M. A., Gould, W. W., Gutierrez, R. G. (2004). An Introduction to survival analysis using STATA (Revised ed.). College Station, TX: State Press.

Fox, J. (2005). The R Commander: A Basic-Statistics Graphical User Interface to R. Journal of Statistical Software, 14(9). http://www.jstatsoft.org/

Hosmer, D. H., Lemeshow, S., \& May, S. (2008). Applied survival analysis: Regression modeling of time-to-event data (2nd ed.). Hoboken. N.J.: Wiley-Interscience.

Jørstad, E. C., Hauer, K., Becker, C., \& Lamb, S. E. (2005). Measuring the psychological outcomes of falling: A systematic review. J Am Geriatr Soc, 53(3), 501-510. http://dx.doi.org/10.1111/j.1532-5415.2005.53172.x

Khodadadi, A. \& Asgharian, M. (2008). Change-point Problem and Regression: An Annotated Bibliography. This working paper is hosted by The Berkeley Electronic Press (bepress) and may not be commercially reproduced without the permission of the copyright holder. http://biostats.bepress.com/cobra/ps/art44

Lawlor, D. A., Patel, R., \& Ebrahim, S. (2003). Association between falls in elderly women and chronic diseases and drug use: Cross sectional study. Bmj, 327(7417), 712-717.

Meng, X.L. (1993). On the absolute bias ratio of ratio estimators. Statistics $\mathcal{E}$ Probability Letters 18: p. 345-348.

Miller, R., Weissman-Berman, D., and Martin, L., "A Predictive model for evidence-based practice in occupational therapy: From a single subject to the world", Research paper presentation, April, 2008, American Occupational Therapy Association Annual Conference.

Powell, L. E., \& Myers, A. M. (1995). The activities-specific balance confidence (abc) scale. The Journals of Gerontology Series A: Biological Sciences and Medical Sciences, 50(1), M28-M34.

Profile of older americans: 2013. (2013). Washington, DC: U.S. Department of Health and Human Services. Department of Health and Human Services.

R Development Core Team (2010). R: A language and environment for statistical computing. Vienna, Austria: R Foundation for Statistical Computing. ISBN 3-900051-07-0, URL http://www.R-Project.org.

R Studio v.0.96. (2014). Downloaded from: www.rstudio.com/ide/docs/release_notes_v0.96

Scheffer, A. C., Schuurmans, M. J., van Dijk, N., van der Hooft, T., \& de Rooij, S. E. (2008). Fear of falling: Measurement strategy, prevalence, risk factors and consequences among older persons. Age and ageing, $37(1), 19-24$.

Schepens, S., Sen, A., Painter, J. A., \& Murphy, S. L. (2012). Relationship between fall-related efficacy and activity engagement in community-dwelling older adults: A meta-analytic review. The American Journal of Occupational Therapy, 66(2), 137-148.

Stevens, J. (2010). A cdc compendium of effective fall interventions: What works for community-dwelling older adults. (2nd ed.) Atlanta, GA: Centers for Disease Control and Prevention, National Center for Injury Prevention and Control, Division of Unintentional Injury Prevention Retrieved from http://www.cdc.gov/HomeandRecreationalSafety/Falls/compendium/0.1_acknowledgements.html.

Stevens, J. A., Corso, P. S., Finkelstein, E. A., \& Miller, T. R. (2006). The costs of fatal and non-fatal falls among older adults. Injury Prevention Journal of the International Society for Child and Adolescent Injury Prevention, 12(5), 290-295. http://dx.doi.org/10.1136/ip.2005.011015

Talley, K. M. C., Wyman, J. F., Gross, C. R., Lindquist, R. A., \& Gaugler, J. E. (2014). Change in balance confidence and its associations with increasing disability in older community-dwelling women at risk for falling. Journal Of Aging And Health, 26(4), 616-636. http://dx.doi.org/10.1177/0898264314526619

Tinetti, M. E., De Leon, C. F. M., Doucette, J. T., \& Baker, D. I. (1994). Fear of falling and fall-related efficacy in relationship to functioning among community-living elders. Journal of Gerontology, 49(3), M140-M147. 
Tinetti, M. E., Richman, D., \& Powell, L. (1990). Falls efficacy as a measure of fear of falling. Journal of gerontology, 45(6), P239-P243. http://dx.doi.org/10.1093/geronj/45.6.P239

Weissman-Miller, D. (2010). A Novel Semiparametric Ratio estimator: A Key to Predicting Long-Term Weight Loss in Obesity. Joint Statistical Meeting (JSM) Proceedings, Non-Parametric Section, Vancouver, B.C.

Weissman-Miller, D., \& Miller, R.J. "New Single-Subject and Small-Group Design: Example, Estimating Weight Loss in Obesity", Research paper presentation, April, 2011, American Occupational Therapy Association Conference.

Weissman-Miller, D., Shotwell, M.P., \& Miller, R.J., (2012). New single-subject and small-n design in occupational therapy: Application to weight loss in obesity. American Journal of Occupational Therapy, 66, 455-462.

Weissman-Miller, D. (2013). Novel point estimation from a Semiparametric Ratio Estimator (SPRE): Long-term health outcomes from short-term linear data, with application to weight loss in obesity. International Journal of Biostatistics, 9(2): 175-184.

Weissman-Miller, D., Graham, K. (2014) Novel Scale Development for Fear of Falling and Falls: Analysed using a Semiparametric Ratio Estimator (SPRE). Joint Statistical Meeting (JSM) Proceedings, Non-Parametric Section, Boston, MA.

\section{Copyrights}

Copyright for this article is retained by the author(s), with first publication rights granted to the journal.

This is an open-access article distributed under the terms and conditions of the Creative Commons Attribution license (http://creativecommons.org/licenses/by/3.0/). 